\title{
Photoluminescent Carbon Dot-Intercalated Bentonite: A Light Responsive Nanohybrid for Lead Removal
}

\author{
Khouloud Jlassi ${ }^{*}$, Abdelgalil Khalaf Ahmed ${ }^{2}$, Hafsa Mutahir ${ }^{2}$, Mostafa H. \\ Sliem $^{1}$, Aboubakr M. Abdullah ${ }^{1 *}$, Mohamed M. Chehimi $^{3,{ }^{*}}$, Igor Krupa $^{1}$
}

1. Center for Advanced Materials, Qatar University, P.O. Box 2713, Doha, Qatar

2. College of Arts and Sciences, Qatar University, P.O. Box 2713, Doha, Qatar

3. Université de Paris, ITODYS, UMR CNRS 7086, 15 rue JA de Baïf, 75013 Paris, France

\author{
Abdelgalil Khalaf Ahmed : aa1305883@student.qu.edu.qa \\ Hafsa Mutahir : $\underline{\text { hm1708761@ @student.qu.edu.qa }}$ \\ Mostafa H. Sliem: mostafa@qu.edu.qa
}

*Correspondence:

khouloud.jlassi@qu.edu.qa (KJ) ; abubakr2@yahoo.com (AMA); and mmchehimi@yahoo.fr $(M M C)$

\begin{abstract}
This work describes the preparation of novel fluorescent bentonite clay (BP), modified with carbon quantum dots nanomaterials (CDs) and its usage as lead removal platform. The CDs were prepared using graphitic waste serving as carbon source material via hydrothermal method, and the as obtained CDs were found to be fluorescent, in spherical shape, positively charged and smaller than $5 \mathrm{~nm}$. Encouraged by their structure and photoluminescence features, they were used hereafter as intercalants or surface modifiers, in order to make fluorescent bentonite nanocomposites. Bentonite was used as negatively charged model of aluminosilicate and reacted with the positively charged CDs. Interestingly, CDs were found to intercalate bentonite as judged from XRD patterns and TEM images. Indeed, the basal distance of bentonite clay d(001) shifted from $1.2 \mathrm{~nm}$ to $2.9 \mathrm{~nm}$, after bentonite modification using the prepared carbon dots; moreover the XRD pattern of BP-CDs, recorded in the some regions, show some additional diffraction peaks along with those for bentonite. The peaks centered at $2 \Theta=27$ degree allocated to the facets of graphitic-like carbon,
\end{abstract}


originated from the carbon dots intercalated in bentonite galleries. The prepared materials were characterized by XPS, FTIR and fluorescence analysis. The obtained results indicate the CDs intercalated inside bentonite matrix were stable over time. The BP-CD nanocomposites were finally used as efficient hybrid platform for led removal with and extraction efficiently of $95 \%$ under light condition, room temperature, in alkaline medium and after only $10 \mathrm{~min}$ of reaction. Thee adsorption process was described using pseudo-second-order kinetics and Langmuir isotherm models, the maximum adsorption capacity, was equal to $400 \mathrm{mg} / \mathrm{g}$ toward $\mathrm{Pb}^{2+}$ removal, at room temperature and $\mathrm{pH}=8$, under light conditions.

To sum up, we have designed UV light stimuli response carbon dot-intercalated clay with high adsorption capacity of $\mathrm{Pb}^{2+}$ and exhibiting long term stability.

\section{Keywords:}

Carbon quantum dots, Bentonite, Hydrothermal process, heavy metal removal

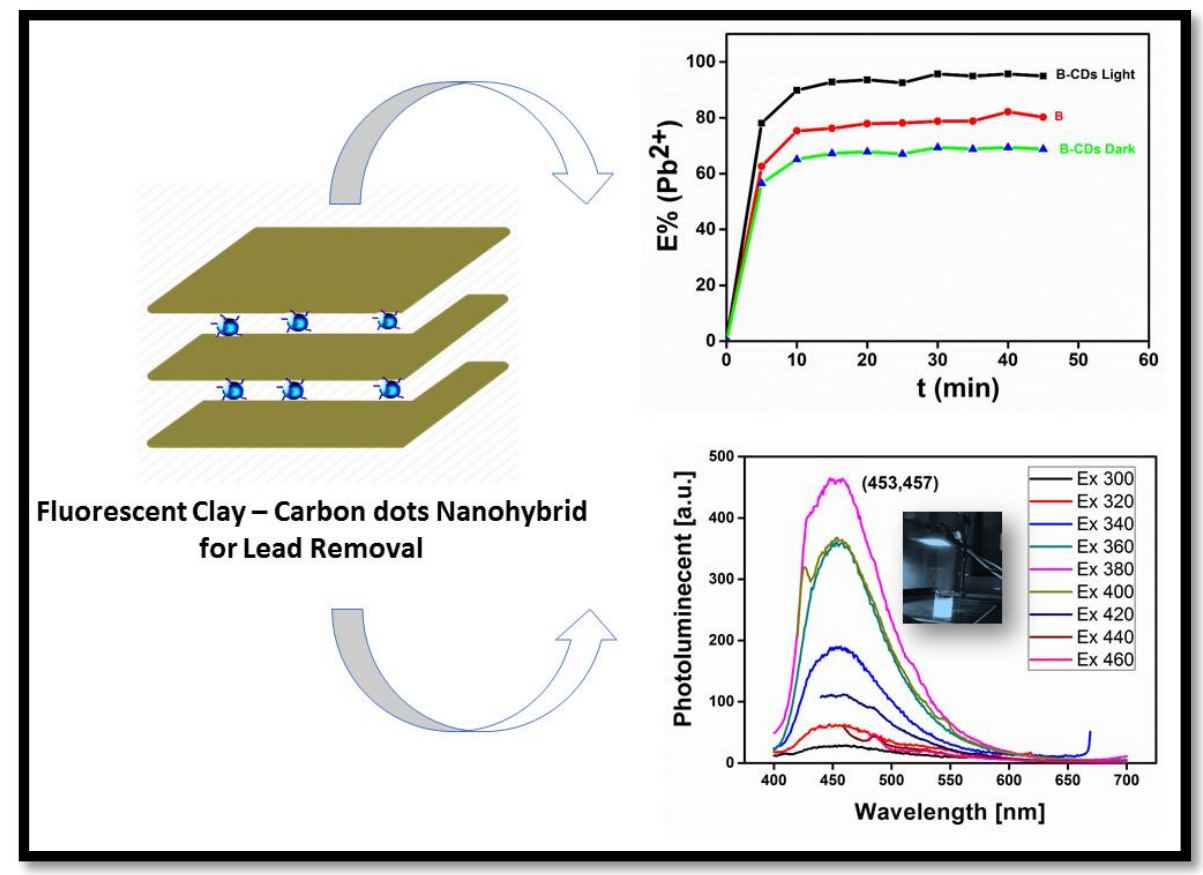

\section{Graphical Abstract}




\section{Introduction}

Bentonite is a layered aluminosilicate of substantial interest among 2D nanomaterials due to its abundance, low cost, high surface area, layered structure, anionic nature with excellent cation exchange capacity, and chemical reactivity (e.g. silanization, arylation). Bentonite is employed in a variety of emergent domains and applications, namely in catalysis, water treatment for heavy metal removal and energy storage. Although bentonite has appealing features, one could take advantage of its reactivity to finely tune its final properties whether it is considered alone or in bentonite-based composites. For example, bentonite clays could be modified using polymers, nucleic acids, surfactants, silanes and diazonium salts in order to turn it organophilic and to tailor interfaces/interphases in bentonite-based composites with a result of outstanding performances (e.g. selective adsorption, sensing, mechanical reinforcement). ${ }^{1}$ Of relevance to the present work, bentonite clay was modified with carbon nanomaterials such as graphite, ${ }^{2}$ graphene ${ }^{3-4}$ and multiwalled carbon nanotubes ${ }^{5-6}$; the resultant hybrid materials were widely used in renewable energy, electronics and environment remediation applications. The resulting hybrids combine both high surface area, adsorptive performance of the 2D nanoclays and high active sites, electric performance of carbon nanomaterials; this synergetic combination was expected to promote the performance of the targeted composites, especially in environmental domain. To the best of our knowledge, no study has been directed towards the preparation of natural clay intercalated with carbon quantum dots. This is an important step in the design layered aluminosilicate/carbon nanomaterial as carbon quantum dots have the unique feature to be fluorescent, which is not the case of CNTs and graphene. Hence, one could use CD-intercalated clay as unique light-responsive material.

Herein, we report a novel and easy way to prepare a fluorescent and hybrid, bentonite-modified with carbon quantum dots via hydrothermal process, the latter was prepared from graphitic waste, and the bentonite served as host matrix, to support, avoid the agglomeration and preserve the fluorescent properties of the as prepared carbon quantum dots. The resulting material is tested as hybrid platform for lead removal. 


\section{Materials and Methods}

\subsection{Chemicals and materials}

Graphite wastes were collected from Qatalum (Doha, Qatar). $\mathrm{H}_{2} \mathrm{SO}_{4}, \mathrm{HNO}_{3}$, ammonia and bentonite nanoclay were purchased from Sigma-Aldrich (Munich, Germany).

\subsection{Synthesis of CDs, B-CDs}

In order to obtain nitrogen dopped carbon quatum dots (CDs), $4 \mathrm{~g}$ of graphite waste was initially grinded then dissolved in sulfuric and nitric acids under stirring/refluxing, as previously described $^{7}$, the as obtained CDs was introduced to bentonite clay(BP) suspension, in order to obtain the (BP-CDs )nanocomposite through cation exchange reaction, 1g of BP (negatively charged) was mixed with 20 wt.\% of CDs (positively charged), the mixture was kept under mechanical stirring at room temperature for $24 \mathrm{~h}$, centrifuged, washed and dried at $60^{\circ} \mathrm{C}$. The as prepared material (B-CDs) was used hereafter as hybrid platform for heavy metal removal, heater for led removal.

\subsection{Adsorption experiment}

The adsorption of BP and BP-CDs was followed by using batch technique. Adsorption processes for single-factor experiments were using $0.01 \mathrm{~g}$ of BP and BP-CDs nanomaterials and $25 \mathrm{~mL}$ of led solution (30 ppm), placed on an orbital shaker at $180 \mathrm{rpm}$ and shaken between five and 45 min until the equilibrium was reached.

The extraction efficiency of heavy metals was calculated using equation (1):

$$
\% \mathrm{E}=\left(\mathrm{C}_{0}-\mathrm{C}_{\mathrm{e}} / \mathrm{C}_{0}\right) \times 100
$$

$\mathrm{C}_{0}$ and $\mathrm{Ce}$; represents the initial and remaining concentration of heavy metal respectively.

The maximum adsorption capacity of lead was calculated, using equation (2):

$$
\mathrm{Qe}=\left(\mathrm{C}_{0}-\mathrm{Ce}\right) \times \mathrm{V} / \mathrm{m} \quad(2)
$$

$\mathrm{C}_{0}$ and $\mathrm{Ce}$ represent, the initial and equilibrium concentration of lead in $(\mathrm{mg} / \mathrm{l}), \mathrm{V}$ is the total volume of solution in (L), and $\mathrm{m}$ in the weight of the used adsorbent in (mg).

In order to study the adsorption kinetics, the Pseudo first and second order models were used respectively, as described below $(3,4)$ : 


$$
\begin{aligned}
& \log \left(q_{e}-q_{t}\right)=\log q_{e}-k_{1} t / 2.303 \\
& 1 /\left(q_{e}-q_{t}\right)=1 / q_{e}+k_{2} t
\end{aligned}
$$

Langmuir and Freundlich models (equations 5 and 6) were used to study the adsorption isotherms and to evaluate the capacity of adsorbent toward heavy metals.

$$
\begin{gathered}
1 / q_{e}=\left(1 / q_{m} K_{L}\right) 1 / C e+1 / q_{m} \\
\log q_{e}=\log k_{F}+1 / n \log C_{e}
\end{gathered}
$$

Where $\mathrm{C}_{\mathrm{e}}, \mathrm{q}_{\mathrm{e}}, \mathrm{q}_{\mathrm{m}} \cdot \mathrm{k}_{\mathrm{L}}$ and $\mathrm{K}_{\mathrm{F}}$, represent the concentration of metal ions at equilibrium $(\mathrm{mg} / \mathrm{L})$, quantity of metal ion $(\mathrm{mg} / \mathrm{g})$, the adsorption capacity $(\mathrm{mg} / \mathrm{g})$ and Langmuir and freundlich equilibrium constant $(\mathrm{L} / \mathrm{mg})$, respectively.

\subsection{Characterizations}

The BP, CDs and B-CDs prepared materials were analyzed using transmission electron microscope (TEM, TecnaiG220, FEI, Hillsboro, OR, USA), X-ray photoelectron spectroscopy (XPS) measurements were conducted using a Kratos Shimadzu Axis Ultra DLD machine (Kratos, Manchester, UK) equipped with a monochromatic Al $\mathrm{K} \alpha$ radiation source $(1486.6 \mathrm{eV})$ under a UHV environment (ca. 5x10-9 Torr). The X-ray diffraction pattern (XRD) was investigated on an X-ray diffractometer (X`Pert-Pro MPD, PANalytical Co., Almelo, Netherlands) using Cu Ka Xray source $(\lambda=1.540598 \AA)$. The Fourier transform infrared spectra were characterized on a Thermo Nicolet Nexus 670 FTIR spectrometer (Thermo Scientific, Madison, WI, USA), and to investigate the optical properties, sample characterization was carried out on a Shimadzu UV3600 spectrophotometer, FLS920 P fluorescence spectrometer (Edinburgh Instruments).

\section{Results and discussion}

\subsection{Preparation of CDs and B-CDs}

The new carbon dots (CDs) were prepared from graphitic waste, via hydrothermal treatment in the presence of ammonia. This process yields outstanding photo-luminescent, monodispersed carbon dots with an average size smaller than 5nm (Fig 1a,b). The as obtained CDs are nitrogen doped, and exhibit unprecedented photo luminescent properties. CDs were used to make 
fluorescent bentonite clay, via hydrothermal process (Fig 1b,c); the bentonite clay was used hereafter as hybrid platform for heavy metal removal.

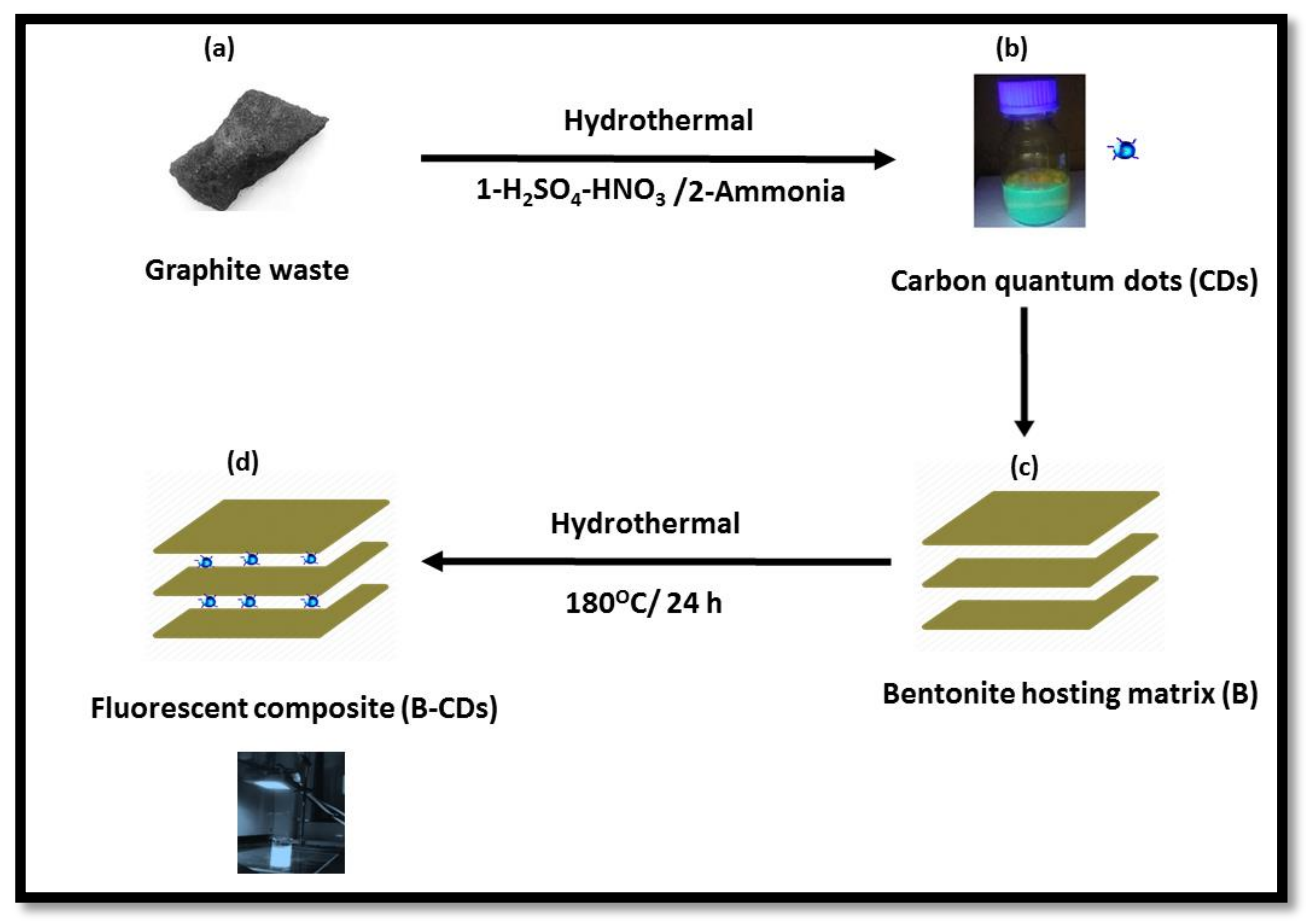

Fig. 1. Upper panel (a) and (b) showing the sequential steps of CDs preparation; lower panel (c) and (d) showing the surface modification of bentonite using the fluorescent CDs via hydrothermal process, in order to obtain fluorescent bentonite (B-CDs).

TEM images (Fig. 2a) revealed spherical and well dispersed nitrogen-doped CDs, with an average diameter of $5 \mathrm{~nm}$. From the corresponding X-ray diffraction (XRD) pattern of B and BCDs (Fig. 2d) and applying the Bragg equation, the basal distance of pure bentonite was found to be equal to $1.3 \mathrm{~nm}$, after cation exchange reaction with the negatively charged CDs, the basal distance was found to increase up to $2.9 \mathrm{~nm}$, indicating a successful cation exchange reaction, between the positively charged clay and CDs. The interlayer distance of BP and B-CDs was investigated as well using TEM images $\mathrm{m}$, and the average distance was calculated trough 2 selected regions, and was found to match the distances deduced from XRD (Figs. $2 b$ and $2 c$ ). XPS was employed to investigate the electronic configuration and surface composition BP, CDs and BP-CDs, as the survey spectra permit to pathway the most important changes in the surface 
composition of CDs and BP surface after and before modification, BP-CDs exhibit intense , N 1s and $\mathrm{C} 1 \mathrm{~s}$ relative to $\mathrm{A} 12 \mathrm{p}$ and $\mathrm{Si} 2 \mathrm{p}$ peaks from bentonite (Fig. 3a).

\subsection{Morphology}

Fig. 2 shows TEM of CDs, BP and BP-CDs. Fig 2.a shows a spherical CDs with an average size smaller than $5 \mathrm{~nm}$, as previously reported ${ }^{8}$. Fig 2.b shows the layered structure of bentonite clay, the basal distance from three selected regions equals to $\sim 1.2 \mathrm{~nm}$, Fig $2 \mathrm{c}-\mathrm{d}$ shows the intercalated clay layers in the presence of well-dispersed carbon dots; the average basal distance of bentonite clay was found to be equal to $2.8 \mathrm{~nm}$.

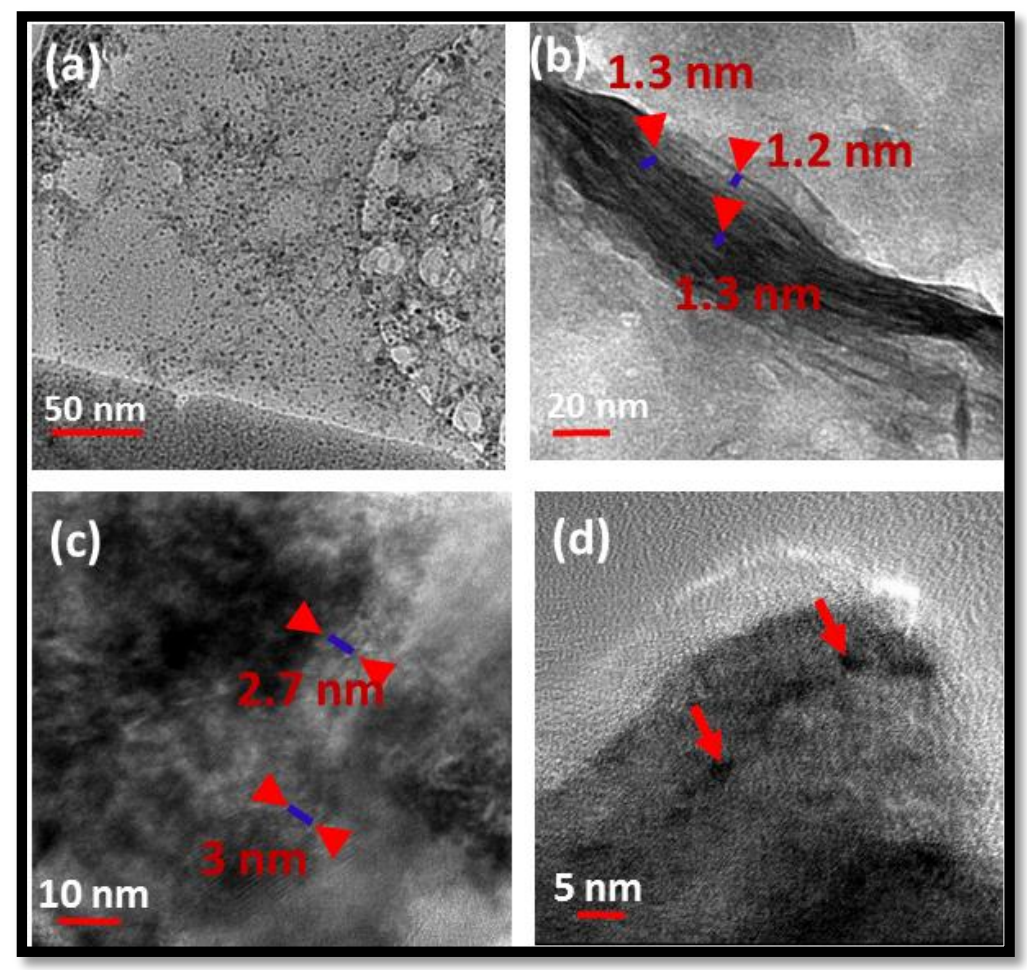

Fig. 2. (a), (b), (c) and (d) TEM of BP, CDs and BP-CDs respectively and at different magnifications.

\subsection{Chemical structure}

XPS analysis was conducted in order to investigate, surface composition of BP-CD nanocomposite and reference materials. Fig. 3a displays survey spectra of B-CDs and CDs that 
confirm the presence of more intense $\mathrm{C} 1 \mathrm{~s}$ and N1s peaks for the BP-CDs nanocomposite indicating modification of the clay by CDs.

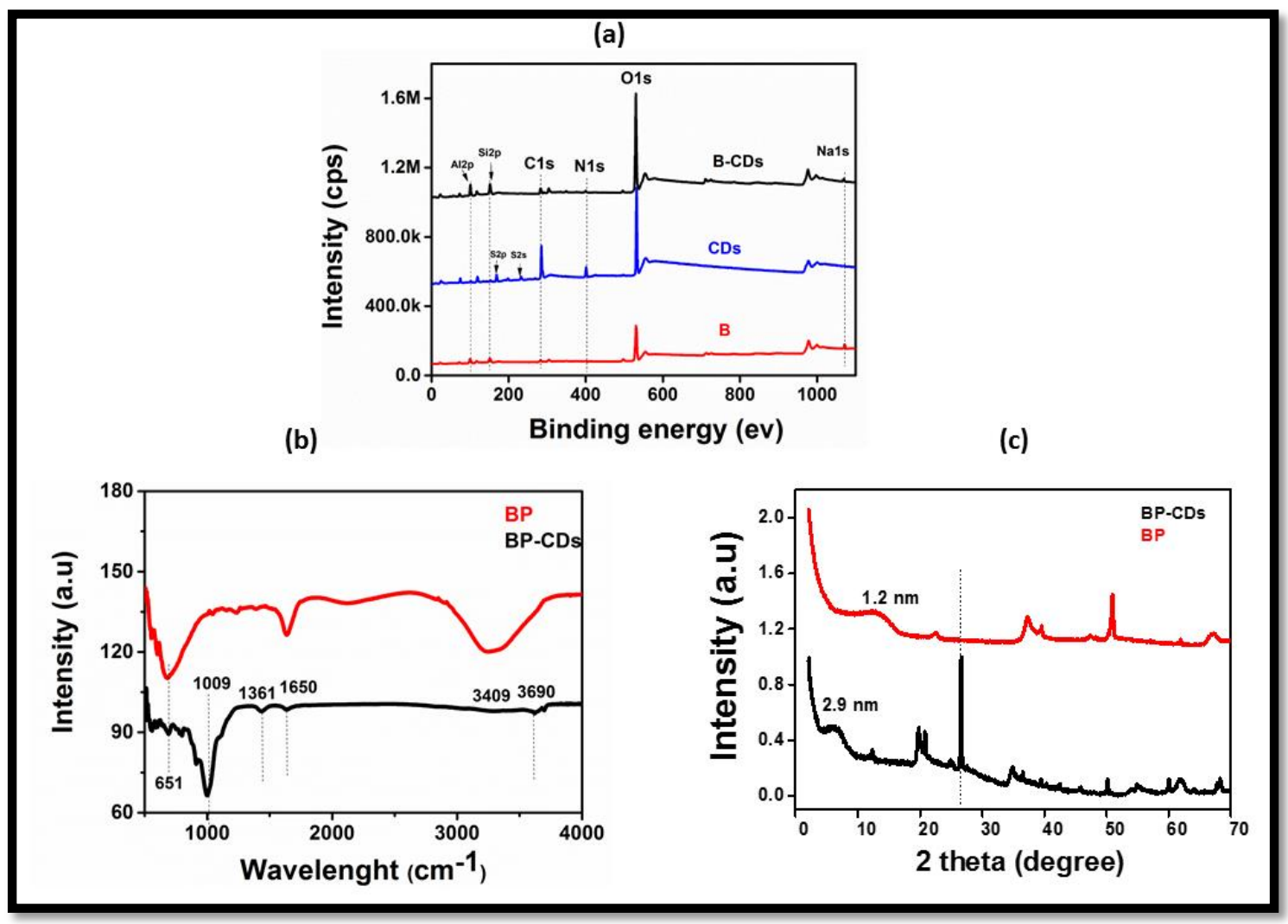

Fig. 3. (a) XPS survey spectra and bentonite, CDs and their nanocomposite BP-CD. (b)FTIR spectra of BP and BP-CDs and (c) XRD patterns of BP and B-CDs nanohybrids.

Table 1. XPS-determined apparent surface composition of BP, CDs and BP-CDs.

\begin{tabular}{|l|l|l|l|l|l|l|l|l|}
\hline Materials & Si & Al & $\mathbf{O}$ & Na & $\mathbf{C}$ & $\mathbf{N}$ & $\mathbf{S}$ & $(\mathbf{C + N}) /(\mathbf{A l + S i})$ \\
\hline BP & 19.5 & 8.30 & 64.6 & 0.72 & 6.95 & - & - & 0.25 \\
\hline CDs & 1.5 & 8 & 39.9 & - & 39.4 & 7.3 & 3.9 & 4.92 \\
\hline BP-CDs & 15.65 & 7.18 & 65 & 0.12 & 10.5 & 1.7 & & 0.53 \\
\hline
\end{tabular}

The chemical compositions of the prepared nanomaterials were reported in Table 1. The atomic ratios $\mathrm{C} / \mathrm{N} / \mathrm{O}$ were determined to be 10.5/1.7/65 for $\mathrm{BP}-\mathrm{CDs}$, whereas for $\mathrm{CDs}$ and $\mathrm{BP}$ the ratio is 
equal to $39.4 / 7.3 / 39.9$ and 6.95/0/64.6, respectively. The detection of nitrogen indicates is due to doping of the prepared CDs, which implies that bentonite is loaded with $\mathrm{N}$-doped CD as judged from the surface elemental composition of BP-CD. The $(\mathrm{C}+\mathrm{N}) /(\mathrm{Al}+\mathrm{Si})$ could serve as an overall chemical descriptor of the intercalation of CDs in bentonite; this descriptor is two-fold higher for BP-CD compared to the purified bentonite BP (see Table 1). One can also note the decrease in the atomic percent of sodium on going from $\mathrm{BP}$ to $\mathrm{BP}-\mathrm{CD}$, which is in line with modification of the clay by intercalation of the quantum dots.

FTIR spectra are shown in Fig. $3 \mathrm{~b}$ for bentonite before and after modification with CDs. It is important to note the decrease of $\mathrm{OH}$ bands intensity, situated at 3413 and $1637 \mathrm{~cm}^{-1}$ reflecting the intercalation of the CDs with the clay surface. Moreover, peaks situated at 651 and $1009 \mathrm{~cm}^{-1}$, could be associated with $\mathrm{Si}-\mathrm{O}-\mathrm{Si}, \mathrm{Mg}-\mathrm{O}-\mathrm{Si}$ vibrations ${ }^{9-10}$; the peak at $1361 \mathrm{~cm}^{-1}$ for BP-CDs material is associated with C-O vibration bonds, and the one at $1457 \mathrm{~cm}^{-1}$ is assigned to $\mathrm{C}-\mathrm{N}$ bonds. Moreover, the presence $\mathrm{C}=\mathrm{C} / \mathrm{C}=\mathrm{O}$ bonds could be detected through the band centred at $1650 \mathrm{~cm}^{-1}$, while the band at $3409 \mathrm{~cm}^{-1}$ indicates the presence of $\mathrm{O}-\mathrm{H}$ and $\mathrm{N}-\mathrm{H}$ groups in BPCDs material. ${ }^{11}$

XRD patterns of BP, CDs and BP-CDs are shown in Fig. 3c. The typical diffraction peaks related to bentonite shift to smaller angles after the modification with CDs, as concluded from Bragg equation. The basal distance of bentonite clay shifted from 1.2 to $2.9 \mathrm{~nm}$ for BP-CDs respectively, in the same range of the basal distances concluded from TEM images (Fig. 2b), whereas XRD pattern of BP-CDs recorded in the some regions show some additional diffraction peaks along with those for bentonite. The peaks centered at $2 \Theta=27^{\circ}$ allocated to the facets of graphitic-like carbon. These TEM and XRD results account for the intercalation of CDs into the bentonite matrix.

\subsection{Optical properties of CDs}

The UV-Vis absorption of B-CDs nanocomposite (Fig. 4a), displays two absorption peak centered at 380 and $450 \mathrm{~nm}$, assigned to $\pi \rightarrow \pi^{*}$ transition and $\mathrm{n} \rightarrow \pi^{*}$ transition, similar to previously reported carbon quantum dots-based materials ${ }^{7,12}$. 
(a)

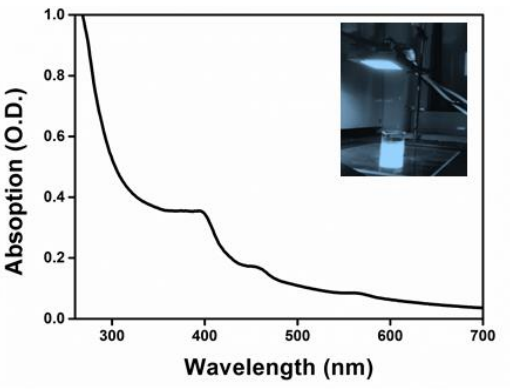

(b)

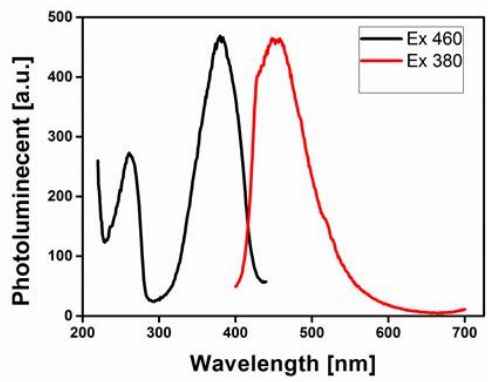

(c)

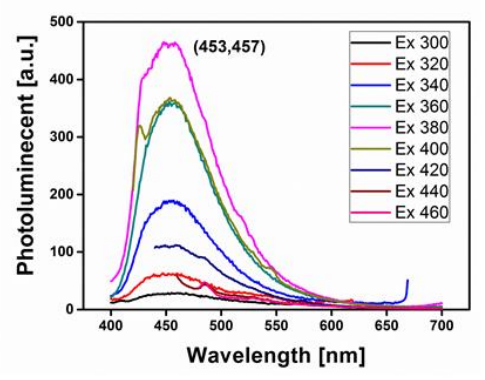

Fig. 4. (a) UV-Vis absorption spectrum, (b) PLE absorption spectrum (c) excitation-dependent emission spectra of CDs at different excitation wavelengths.

The photoluminescence properties of the nanocomposites were also evaluated with the help of photoluminescence spectroscopy. The emission spectra Fig. 4c, of the B-Ds show the increase in the PL intensity as the excitation wavelength is increasing from 300 to $460 \mathrm{~nm}$. The maximum emission intensity reached $454 \mathrm{~nm}$ (blue emission) when it is excited at $460 \mathrm{~nm}$. This phenomena is associated with carbon quantum dots-based nanohybrids ${ }^{13}$. We have checked these optical properties and found them stable over a period of six months, without any significant alteration.

\subsection{Application to the removal of $\mathrm{Pb}^{2+}$ from water}

The removal of $\mathrm{Pb}^{2+}$ from the aqueous solution was evaluated using pure bentonite (B) as well as the prepared nanohybrid material (B-CDs) under dark and light conditions. The selectivity test was evaluated in presence of $\mathrm{Cu}^{2+}, \mathrm{Zn}^{2+}$, and $\mathrm{Co}^{2+}$ at $\mathrm{pH} 8.0$, and the relative extraction efficiency equals 48,33, 66 and $95 \%$, respectively. The removal of the metal ions was assessed using ICP analysis technique. The adsorption performance of $\mathrm{Pb}^{2+}$, using $\mathrm{B}$ and (B-CDs) nanohybrid was also investigated versus $\mathrm{pH}$ and contact time. $\mathrm{B}$ and $\mathrm{B}-\mathrm{CDs}$ were immersed in a 30-ppm solution of $\mathrm{Pb}^{2+}$ up to $45 \mathrm{~min}$, the remaining concentration of $\mathrm{Pb}^{2+}$ was detected using (ICP) analysis technique, as shown in Fig. 5d, the extraction efficiency percentage of $\mathrm{Pb}^{2+}$ was found to increase in alkaline solution, and the highest extraction efficiency percentages $(\mathrm{E}=95 \%)$ were noted in alkaline solution at $\mathrm{pH}=8$ and room temperature Fig. 5e, using BP-CDs under light stimulus, compared to $81 \%$ and $70 \%$ respectively, only in presence of unmodified BP and BP-CDs under dark conditions. In order 
to evaluate the rate of $\mathrm{Pb}^{2+}$ removal on $\mathrm{B}-\mathrm{CDs}$, the kinetics of the sorption model was applied. First-order and pseudo-second-order models (equations 3,4) were used to study the variations in adsorption with time ${ }^{14-15}$. The better correlation coefficient values were noted with pseudosecond-order model compared to the first-order kinetics (Fig. 5c and Table 2). From the above, the dominant mechanism could be chemical adsorption ${ }^{16}$. The adsorption isotherm is applied to estimate the adsorption capacity of an adsorbent, moreover it gives a prediction, and how the material interacts with adsorbents. Toward this end, the Langmuir and Freundlich isotherms (equations 5, 6), were used.
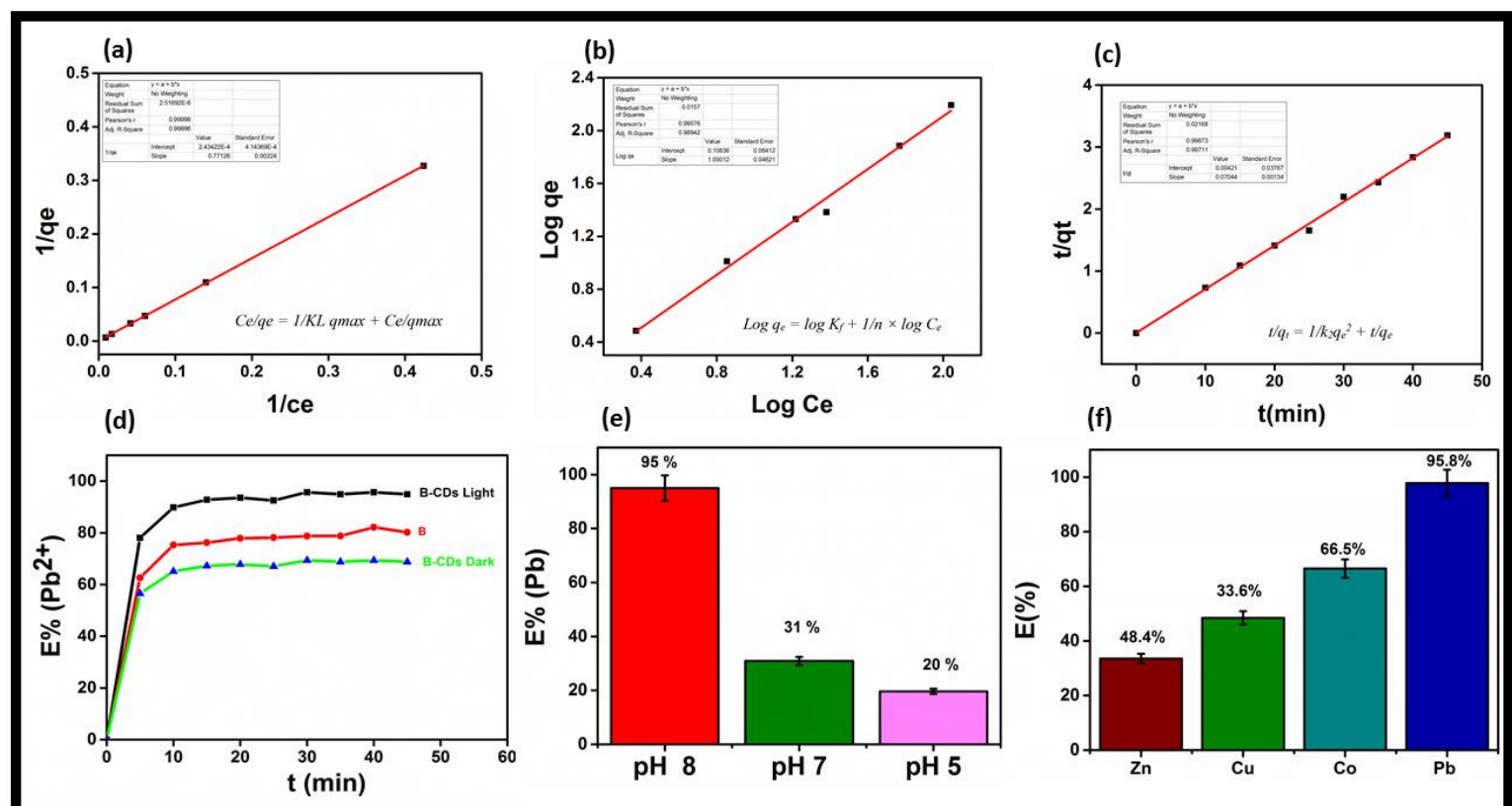

Fig. 5. (a) The fitting results of Langmuir models, (b) Freundlich models, (c) Pseudo-secondorder mode for $\mathrm{Pb}^{2+}$ adsorption using B-CDs under light conditions, (d) Trends of the extraction efficiency percentages for cadmium using BP, BP-CDs under light at $\mathrm{pH}=8$, (e) Trends of the extraction efficiency percentages for lead at different $\mathrm{pH}$, (f) selectivity test.

Langmuir model was better fitted than Freundlich model based on the correlation coefficients $\left(\mathrm{R}^{2}\right)$ from Table 2, Fig. 5a and Fig. 5b, and the maximum adsorption capacity, was equal to $400 \mathrm{mg} / \mathrm{g}$ toward $\mathrm{PB}$ (II) removal, at room temperature and $\mathrm{pH}=8$ and under light conditions. 
Table 2. Rate constants of the pseudo-first-order and pseudo-second-order kinetics models for the adsorption of $\mathrm{Pb}^{2+}$ on $\mathrm{CH}-\mathrm{CDs}$.

\begin{tabular}{|c|c|c|c|c|c|c|c|c|}
\hline \multicolumn{3}{|c|}{ Pseudo-second order } & \multicolumn{3}{|c|}{ Langmuir constants } & \multicolumn{3}{|c|}{ Freundlich constants } \\
\hline $\mathbf{q}_{\mathrm{e}}(\mathrm{mg} / \mathrm{g})$ & $\begin{array}{l}\mathbf{k}_{2}(\mathrm{~g} / \mathrm{mg} \\
\mathrm{min})\end{array}$ & $\mathbf{R}^{2}$ & $\begin{array}{l}\mathbf{K L} \\
(\mathrm{L} / \mathrm{mg})\end{array}$ & $\begin{array}{l}\mathbf{q m} \\
(\mathrm{mg} / \mathrm{g})\end{array}$ & $\mathbf{R}^{2}$ & $\begin{array}{l}\text { Kf } \\
(\mathrm{mg} / \mathrm{g})(\mathrm{mg} / \mathrm{L})^{1 / \mathrm{n}}\end{array}$ & $\mathbf{n}$ & $\overline{\mathbf{R}^{2}}$ \\
\hline 14.2 & 1.18 & 0.99 & $\mathbf{0 . 0 3}$ & 400 & 0.99 & 1.28 & $\mathbf{1 . 0 1}$ & 0.98 \\
\hline
\end{tabular}

La valeur $14.2 \mathrm{mg} / \mathrm{g}$ est faible par rapport à la valeur annoncée de $400 \mathrm{mg} / \mathrm{g}$

\subsection{Comparison with other absorbents}

Table 3 compares the adsorption capacity of $\mathrm{Pb}^{2+}$ using $\mathrm{B}-\mathrm{CD}$ nanohybrid with similar materials previously investigated.

Table 3. Summary of some adsorbent-based carbon quantum dots used for $\mathrm{Cd}^{2+}$ removal

\begin{tabular}{|c|c|c|c|c|}
\hline Adsorbent & $\mathrm{Qm} / \mathrm{mg} \mathrm{g}^{-1}$ & $\begin{array}{l}\text { Experimental } \\
\text { conditions }\end{array}$ & $\mathrm{t} / \mathrm{min}$ & Refs \\
\hline Kaolinite clay modified with polyphosphate & 40 & $\begin{array}{l}\mathrm{pH}=5 \\
25^{\circ} \mathrm{C}\end{array}$ & 60 & 17 \\
\hline Bentonite clay & 26.3 & $\begin{array}{l}\mathrm{pH}=4 \\
\mathrm{~T}=25^{\circ} \mathrm{C}\end{array}$ & 60 & 18 \\
\hline $\begin{array}{l}\text { Diazonium-based ion-imprinted polymer/clay } \\
\text { nanocomposite }\end{array}$ & 301 & $\begin{array}{l}\mathrm{pH}=7 \\
\mathrm{~T}=30^{\circ} \mathrm{C}\end{array}$ & 30 & 19 \\
\hline Magnetic Sepiolite Clay & 96.15 & $\begin{array}{l}\mathrm{pH}=7 \\
\mathrm{~T}=25^{\circ} \mathrm{C}\end{array}$ & 30 & 20 \\
\hline Attapulgite clay modified with $\mathrm{MgO}$ & 127.6 & $\begin{array}{l}\mathrm{pH}=5, \\
\mathrm{~T}=55^{\circ} \mathrm{C}\end{array}$ & 1440 & 21 \\
\hline Bentonite modified with Carbon quantum dots & 400 & $\begin{array}{l}\mathrm{pH}=8 \\
\mathrm{~T}=25^{\circ} \mathrm{C} \\
\mathrm{UV}=365 \mathrm{~nm}\end{array}$ & 45 & This work \\
\hline
\end{tabular}

As shown in Table 3, the B-CDs nanohybrid presents superior adsorption capacity compared to other adsorbents, due to the formation of strong complex between the free carboxylic, $\mathrm{N}$ and sulfur groups present in $\mathrm{CDs}$ and $\mathrm{Pb}^{2+}$ ions from one side ${ }^{7,22}$, and the cation exchange reaction from the other side, 
that may happen between the $\mathrm{Na}^{+}$present in bentonite clay galleries and the $\mathrm{Pb}^{2+}$ metal present in solution as shown in Fig. $6 .^{23-24}$

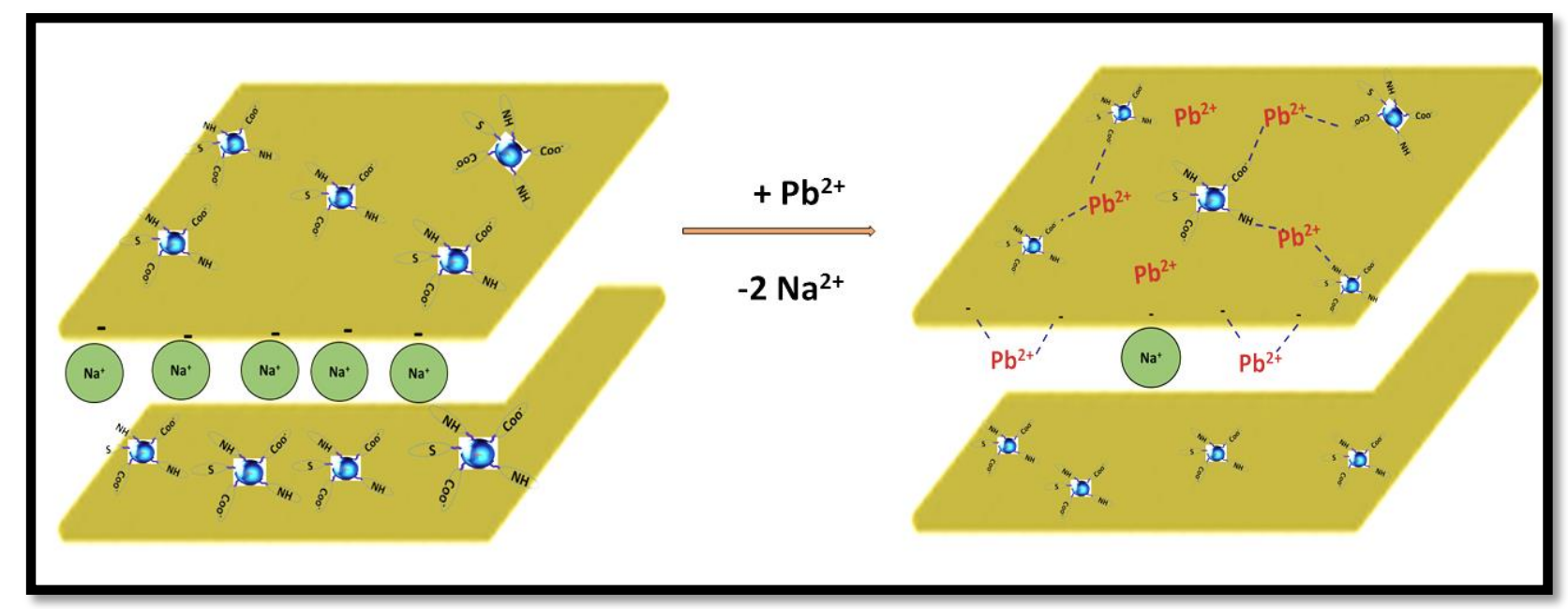

Fig. 6. Schematic illustration of the B-CDs nanohybrid material and potential mechanism of lead removal.

\section{Conclusion}

In conclusion, BP-CDs were successfully prepared via hydrothermal process using CDs from graphitic waste; natural bentonite served as hosting matrix. The CDs were successfully introduced into bentonite nanoclay matrix and form well dispersed BP-CDs, stable and fluorescent. The latter was successfully used as platform for lead removal from water, with and extraction efficiency around $95 \%$. The equilibrium time of adsorption was faster than other reported works (only $10 \mathrm{~min}$ ). Further, the as prepared B-CDs were found to be fluorescent and exhibited wavelength dependent photoluminescence properties. The adsorption process could be described using pseudo-second-order kinetics and Langmuir isotherm models; the maximum adsorption capacity was equal to $400 \mathrm{mg} / \mathrm{g}$ toward PB (II) removal, at room temperature and $\mathrm{pH}=8$, under light conditions. This new fluorescent, long-term stable nanohydrid could thus be qualified as a low-cost, sustainable and green adsorbent for clean water treatment and production sector. 


\section{Acknowledgements:}

This report was made possible by a UREP award [UREP25-091-2-034] from the Qatar National Research Fund (a member of The Qatar Foundation). The statements made herein are solely the responsibility of the authors. The authors appreciate the central laboratories unit, Qatar University, for their assistance in the ICP analysis.

Declaration of interest: The authors declare no conflict of interest

\section{References}

1. Jlassi, K.; Chehimi, M. M.; Thomas, S., Clay-polymer nanocomposites. Elsevier: 2017.

2. Liu, S.; Han, J.; Gao, Q.; Kang, W.; Ren, R.; Wang, L.; Chen, D.; Wu, D., Lauric acid/bentonite/flake graphite composite as form-stable phase change materials for thermal energy storage. Materials Express 2020, 10 (2), 214-224.

3. Chang, Y. S.; Au, P. I.; Mubarak, N. M.; Khalid, M.; Jagadish, P.; Walvekar, R.; Abdullah, E. C., Adsorption of $\mathrm{Cu}$ (II) and $\mathrm{Ni}$ (II) ions from wastewater onto bentonite and bentonite/GO composite. Environmental Science and Pollution Research 2020, 27 (26), 33270-33296.

4. Dhumal, P. S.; Khose, R. V.; Wadekar, P. H.; Lokhande, K. D.; Some, S., Graphenebentonite supported free-standing, flexible membrane with switchable wettability for selective oil-water separation. Separation and Purification Technology 2021, 118569.

5. Huseynov, A.; Israfilov, A.; Mammadova, S.; Abdullayeva, S.; Sokolov, S.; Goryunkov, A.; Guliyev, A., Fabrication and characterization of MWCNT/natural Azerbaijani bentonite electroconductive ceramic composites. Journal of Composite Materials 2019, 53 (26-27), 39093923.

6. Özkan, A., Effect of gold nanoparticle functionalized multi-walled carbon nanotubes on the properties of Na-bentonite water based drilling fluid. Fresenius Environmental Bulletin 2020, 29 (1), 143-151.

7. Jlassi, K.; Eid, K.; Sliem, M. H.; Abdullah, A. M.; Chehimi, M. M.; Krupa, I., Rational synthesis, characterization, and application of environmentally friendly (polymer-carbon dot) hybrid composite film for fast and efficient UV-assisted $\mathrm{Cd} 2+$ removal from water. Environmental Sciences Europe 2020, 32 (1), 1-13.

8. Jlassi, K.; Mallick, S.; Eribi, A.; Chehimi, M. M.; Ahmad, Z.; Touati, F.; Krupa, I., Facile preparation of NS co-doped graphene quantum dots (GQDs) from graphite waste for efficient humidity sensing. Sensors and Actuators B: Chemical 328, 129058.

9. Mudasir, M.; Baskara, R. A.; Suratman, A.; Yunita, K. S.; Perdana, R.; Puspitasari, W., Simultaneous adsorption of $\mathrm{Zn}$ (II) and $\mathrm{Hg}$ (II) ions on selective adsorbent of dithizoneimmobilized bentonite in the presence of $\mathrm{Mg}$ (II) Ion. Journal of Environmental Chemical Engineering 2020, 8 (4), 104002.

10. Jlassi, K.; Eid, K.; Sliem, M. H.; Abdullah, A. M.; Chehimi, M. M., Calix [4] arene-clicked clay through thiol-yne addition for the molecular recognition and removal of $\mathrm{Cd}$ (II) from wastewater. Separation and Purification Technology 2020, 251, 117383. 
11. Dimos, K.; Arcudi, F.; Kouloumpis, A.; Koutselas, I. B.; Rudolf, P.; Gournis, D.; Prato, M., Top-down and bottom-up approaches to transparent, flexible and luminescent nitrogen-doped carbon nanodot-clay hybrid films. Nanoscale 2017, 9 (29), 10256-10262.

12. Liu, Y.; Wu, P.; Wu, X.; Ma, C.; Luo, S.; Xu, M.; Li, W.; Liu, S., Nitrogen and copper (II) codoped carbon dots for applications in ascorbic acid determination by non-oxidation reduction strategy and cellular imaging. Talanta 2020, 210, 120649.

13. Yang, Y.; Wu, D.; Han, S.; Hu, P.; Liu, R., Bottom-up fabrication of photoluminescent carbon dots with uniform morphology via a soft-hard template approach. Chemical Communications 2013, 49 (43), 4920-4922.

14. Khaksar Najafi, E.; Jamshidi Chenari, R.; Arabani, M., Sorption kinetics of lead and zinc ions by clay-fly ash geopolymers. Environmental Engineering Science 2021.

15. Sirajudheen, P.; Karthikeyan, P.; Vigneshwaran, S.; Meenakshi, S., Synthesis and characterization of La (III) supported carboxymethylcellulose-clay composite for toxic dyes removal: Evaluation of adsorption kinetics, isotherms and thermodynamics. International journal of biological macromolecules 2020, 161, 1117-1126.

16. Messaoud-Boureghda, M. Z., Adsorption of heavy metals (cadmium, chrome and copper) on grafted cellulose: Establishment of kinetic models. Proceedings of the International Academy of Ecology and Environmental Sciences 2019, 9 (2), 53.

17. Amer, M. W.; Khalili, F. I.; Awwad, A. M., Adsorption of lead, zinc and cadmium ions on polyphosphate-modified kaolinite clay. Journal of environmental chemistry and ecotoxicology 2010, 2 (1), 001-008.

18. Khan, M.; Hegde, R.; Shabiimam, M., Adsorption of lead by bentonite clay. International Journal of Scientific Research and Management 2017, 5 (7), 5800-5804.

19. Msaadi, R.; Ammar, S.; Chehimi, M. M.; Yagci, Y., Diazonium-based ion-imprinted polymer/clay nanocomposite for the selective extraction of lead (II) ions in aqueous media. European Polymer Journal 2017, 89, 367-380.

20. Fayazi, M., Facile hydrothermal synthesis of magnetic sepiolite clay for removal of $\mathrm{Pb}$ (II) from aqueous solutions. Analytical and Bioanalytical Chemistry Research 2019, 6 (1), 125-136.

21. Huang, R.; Lin, Q.; Zhong, Q.; Zhang, X.; Wen, X.; Luo, H., Removal of Cd (II) and Pb (II) from aqueous solution by modified attapulgite clay. Arabian Journal of Chemistry 2020, 13 (4), 4994-5008.

22. Arancibia-Miranda, N.; Baltazar, S. E.; García, A.; Muñoz-Lira, D.; Sepúlveda, P.; Rubio, M. A.; Altbir, D., Nanoscale zero valent supported by zeolite and montmorillonite: template effect of the removal of lead ion from an aqueous solution. Journal of hazardous materials 2016, 301, 371-380.

23. Oubagaranadin, J. U. K.; Murthy, Z., Adsorption of divalent lead on a montmorilloniteillite type of clay. Industrial \& Engineering Chemistry Research 2009, 48 (23), 10627-10636.

24. Terán, E. J.; Montes, M. L.; Rodríguez, C.; Martino, L.; Quiroga, M.; Landa, R.; Sanchez, R. T.; Pace, D. D., Assessment of sorption capability of montmorillonite clay for lead removal from water using laser-induced breakdown spectroscopy and atomic absorption spectroscopy. Microchemical Journal 2019, 144, 159-165. 